\title{
Deep sequencing reveals complex mechanisms of microRNA deregulation in colorectal cancer
}

\author{
GAOFENG LIANG ${ }^{1,3}$, JUNSHENG LI $^{2}$, BO SUN $^{1}$, SHUCHUN LI $^{1}$, LANXIN LÜ $^{1}$, \\ YUANYUAN WANG ${ }^{1},{\text { BAOAN } \text { CHEN }^{2} \text { and ZHONGDANG XIAO }}^{1}$ \\ ${ }^{1}$ State Key Laboratory of Bioelectronics, School of Biological Science and Medical Engineering, \\ Southeast University, Nanjing 210096; ${ }^{2}$ Department of Hematology and Oncology, Zhongda Hospital, \\ Southeast University, Nanjing 210009; ${ }^{3}$ School of Medical Technology and Engineering, \\ Henan University of Science and Technology, Luoyang, Henan 471003, P.R. China
}

Received February 8, 2014; Accepted April 9, 2014

DOI: $10.3892 /$ ijo.2014.2474

\begin{abstract}
Deregulated microRNAs (miRNAs) have been reported to be of functional relevance for tumor biology. In this study, we analyzed the small RNA transcriptomes of 10 paired colorectal cancer samples using SOLiD next-generation sequencing, and generated a total of $>15141000$ reads. miRNA expression profiles obtained by SOLiD sequencing correlated well with quantitative PCR results. The results showed that the expression of 36 miRNAs was significantly different between the two groups of samples. Additionally, we explored the global miRNA-mRNA interactions using a statistical model. In-depth analysis reveals a diagram of extensive post-transcriptional miRNA regulations. Signaling pathways associated with the miRNA altered expression signature were identified using gene enrichment analysis. The results suggest that these microRNAs, in the aggregate, regulate signaling pathways, such as MAPK, Wnt and p53 pathways in cancer, which are known to be involved in the transformation of colorectal cancer. This evidence demonstrates that miRNAs can cooperatively regulate a given pathway and play a subtle role by regulating their target genes.
\end{abstract}

\section{Introduction}

Colorectal cancer (CRC) is one of the most frequent cancers and a common cause of cancer-related deaths in the world (1). Currently, prognosis for CRC largely relies upon descriptive staging systems depending on the morphology and histopa-

Correspondence to: Professor Zhongdang Xiao, State Key Laboratory of Bioelectronics, Southeast University, Sipailou 2, Nanjing 210096, P.R. China

E-mail: zdxiao@seu.edu

Dr Baoan Chen, Department of Hematology and Oncology, Zhongda Hospital, Southeast University, Nanjing 210009, P.R. China

E-mail: cba8888@hotmail.com

Key words: microRNA, colorectal cancer, deep sequencing, regulation thology of the tumor (2). The alteration of normal cells to malignant carcinomas involves a multi-step process with accumulation of both genetic and epigenetic changes, leading to a temporal activation of oncogenes and inactivation of tumor suppressor genes that confer a selective advantage to cells containing these alterations (3). Most CRC cases have the aberration related to Wnt, KRAS, TGF- $\beta$, $\beta$-catenin and 553 pathways, as result in an accumulated effect on CRC progression (4). This suggests that underlying levels of regulation exist to control the complex cross-talk between different signal transduction pathways.

To better understand the molecular mechanism of colorectal cancer, a number of mRNA expression profiling studies on protein coding genes have been performed to further explore the pathogenesis of CRC $(5,6)$. However, the possibility of mRNA degradation during RNA sample preparation and the post-translational regulation on mRNA translations impair the application of mRNA assay on illustrating the exact gene expression process. Compared to the instability of mRNAs, microRNAs (miRNAs), a newly discovered class of short 22 nucleotide (nt) non-coding RNAs, were paid more and more attentions because of their stability in the samples and the universality in gene regulations $(7,8)$. miRNAs are involved in the regulation of most physiological processes, including differentiation, development and apoptosis (9). In cancer, miRNAs may exert oncogenic function by inhibiting tumor suppressor genes or may act as tumor suppressors by inhibiting oncogenes (10). Bioinformatics analysis suggested that miRNAs may regulate $\sim 30 \%$ of the human protein coding genes, indicating that these small non-coding RNAs may coordinate the interplay between complex signal transduction pathways (11).

Several groups have analyzed miRNA expression in colorectal cancer using miRNA microarrays or highthroughput RT-qPCR. They reported broad deregulation of miRNA expression patterns (12-15). Further functional analysis identified that the dysregulated miR-135 family and miR-17 family are important for proliferation, migration as well as invasive growth of colorectal cancer cells $(16,17)$. Subsequently, a small number of clinical samples have been screened using a deep sequencing approach to investigate 
the differentially expressed miRNAs identifying novel miRNAs (18). Notwithstanding these earlier studies in CRC, there still remains the need to confirm earlier observations in a large set of clinical CRC samples and to examine comprehensively the molecular mechanism of altered miRNA expression in this cancer.

In this study, we compared the small RNA transcriptomes of 10 colorectal cancer samples versus adjacent normal tissues by means of ultra-deep sequencing using SOLiD system (Applied Biosystems, USA). The results were confirmed by RT-qPCR. Subsequently, we investigated the miRNAmRNA relationships using correlation analysis and applied a statistical model to infer the regulatory relationships between mRNA and miRNAs. This is a comprehensive presentation of the composition of the small RNA transcriptome in a kind of primary tumor, using colorectal cancer as a model system, which provided insights into the heterogeneity of the small RNA transcriptomes in cancer. Our results also present a unique perspective on miRNA regulation mechanism by predicting causal regulators of miRNAs and the downstream mRNA targets of miRNAs.

\section{Materials and methods}

Sample preparation and RNA isolation. Twenty fresh-frozen human colorectal cancer samples in late stage were obtained from Affiliated ZhongDa Hospital (Southeast University, China), including 10 colorectal cancer samples and 10 adjacent normal tissues. Written informed consent was obtained from all patient representatives, and this work obtained ethics approval from the ethics committee of Zhongda Hospital. The median tumor content of all CRC samples was $>80 \%$, without significant difference in tumor content between these samples. Samples were divided into two groups (tumor group and adjacent normal tissues), treated with TRIzol (Invitrogen, Carlsbad, CA, USA), and then all 10 samples were pooled, respectively, and small RNA was extracted using the mirVana miRNA isolation kit (Ambion, Austin, USA) according to the manufacturer's recommendations. RNAs were quantified using oneDrop ${ }^{\circledR}$ ND-1000 and quality was tested by migration of $200 \mathrm{ng}$ RNA on $1 \%$ agarose gel.

Small RNA preparation and SOLID sequencing. Small RNA for deep sequencing prepared was converted into a cDNA library according to the manufacturer's protocol [SREK (small RNA expression kit), Applied Biosystems]. Template bead preparation, emulsion PCR and deposition were performed according to the standard protocol, and slides were analyzed on a SOLiD system (Applied Biosystems). The results of SOLiD sequencing was presented in the form of nucleotide sequences and their coverage. Two mismatches for read lengths between 12 and 14 and three mismatches for longer reads were allowed. Raw expression values (read counts) were obtained by comparing with Genebank, Human Genome RefSeq, miRBase release 15.0, and registered miRNAs could be screened out (the new data were deposited in GenBank, NCBI tracking system \#16137685).

The differentially expressed miRNAs in colorectal cancer were performed with fold-change analysis (19). The coverage of a miRNA in the miRNA group was calculated with formula 1:

$$
\text { Coverage }_{\text {miRNA }}=c_{\text {miRNA }} / C_{\text {miRNA }}
$$

In which $\mathrm{c}_{\text {miRNA }}$ means the reads of a special $\mathrm{m}_{\mathrm{iRNA}}$ and $\mathrm{C}_{\text {miRNA }}$ means the reads of all miRNAs in a sample. Therefore, the fold-change of miRNA expression was expressed in formula 2 below:

Fold $_{\text {miRNA }}=\frac{\left(\text { Coverage }_{\text {miRNA }}\right)_{\text {test }} \times 10^{6}}{\left(\text { Coverage }_{\text {miRNA }}\right)_{\text {control }} \times 10^{6}}=\frac{\left(c_{\text {miRNA }} / C_{\text {miRNA }}\right)_{\text {test }} \times 10^{6}}{\left(c_{\text {miRNA }} / C_{\text {miRNA }}\right)_{\text {control }} \times 10^{6}}$

Quantitative $R T-P C R$. To verify the differentially expressed miRNAs in colorectal cancer, we randomly selected 16 miRNAs and performed quantitative RT-PCR using stemloop primers and the ABI 7500 System (Applied Biosystems). For each sample tested, retrotranscription using specific reverse transcription primers were performed on $1 \mu \mathrm{g}$ of total RNAs according to manufacturer's protocol. U6 snRNA was used for the normalization of expression data. The relative amount of miRNAs and U6 was determined using the $2^{-\Delta \Delta \mathrm{CT}}$ method (20), where $-\Delta \Delta \mathrm{CT}=\left(\mathrm{CT}_{\text {target }}-\mathrm{CT}_{\mathrm{U} 6}\right)_{\text {sample }}-\left(\mathrm{CT}_{\text {target }}-\mathrm{CT}_{\mathrm{U} 6}\right)_{\text {calibrator }}$. Briefly, for the validation set of samples, expression was normalized with U6 and with corresponding normal colon sample. The reactions were incubated in a 96-well plate at $95^{\circ} \mathrm{C}$ for $10 \mathrm{~min}$, followed by 40 cycles of $95^{\circ} \mathrm{C}$ for $15 \mathrm{sec}$, and $60^{\circ} \mathrm{C}$ for $1 \mathrm{~min}$. All RT-qPCR reactions included a no template control were performed in triplicate.

Target gene prediction. PicTar Web interface (http://pictar. mdc-berlin.de/cgi-bin) was used for miRNA target prediction. This tool performs a prediction of miRNA targets based on a perfectly Watson-crick-base-paired stretch of $\sim 7 \mathrm{nt}$ starting at either the first or the second base of the miRNA. Initially, a predicted target gene cluster was generated by PicTar from all expressed miRNAs. All predicted target genes for miRNAs and PicTar score were extracted in order to determine mRNA inhibitor ratio for next step.

miRNA repression effect on $m R N A s$. The degree of miRNA repression effecton a specific mRNAk was calculated with formula 3:

$$
R_{\text {mRNA }_{k}}=\left[\sum_{i=1}^{l}\left(\text { Count }_{m i R N A_{i}} / n_{i j}\right) * \text { weight }\right] / N_{\text {total }}
$$

In which, $\mathrm{R}_{\text {mRNAk }}$ means the repression degree (RD) to the mRNA $_{k}$ by its target miRNAs; Count $_{\text {miRNAi }}$, the amount of the $i$ th miRNA; $i$, the number of miRNAs target to the mRNA , $i=1,2, \ldots, n ; n_{i j}$, the number of mRNA targeted by the miRNAs; weight, the weight coefficient for the repression degree to the mRNA $_{k}$ by its target miRNAs, which is the PicTar score for interaction of miRNA with its target mRNAs; $N_{\text {total }}$, the number of all miRNAs detected in the sample.

Bioinformatics analysis. Based on the results from formula 3, gene ontology (GO) enrichment analysis of biological processes (hierarchical level 3,4 and 5) was applied on this set of predicted miRNA target genes, in which human genome 

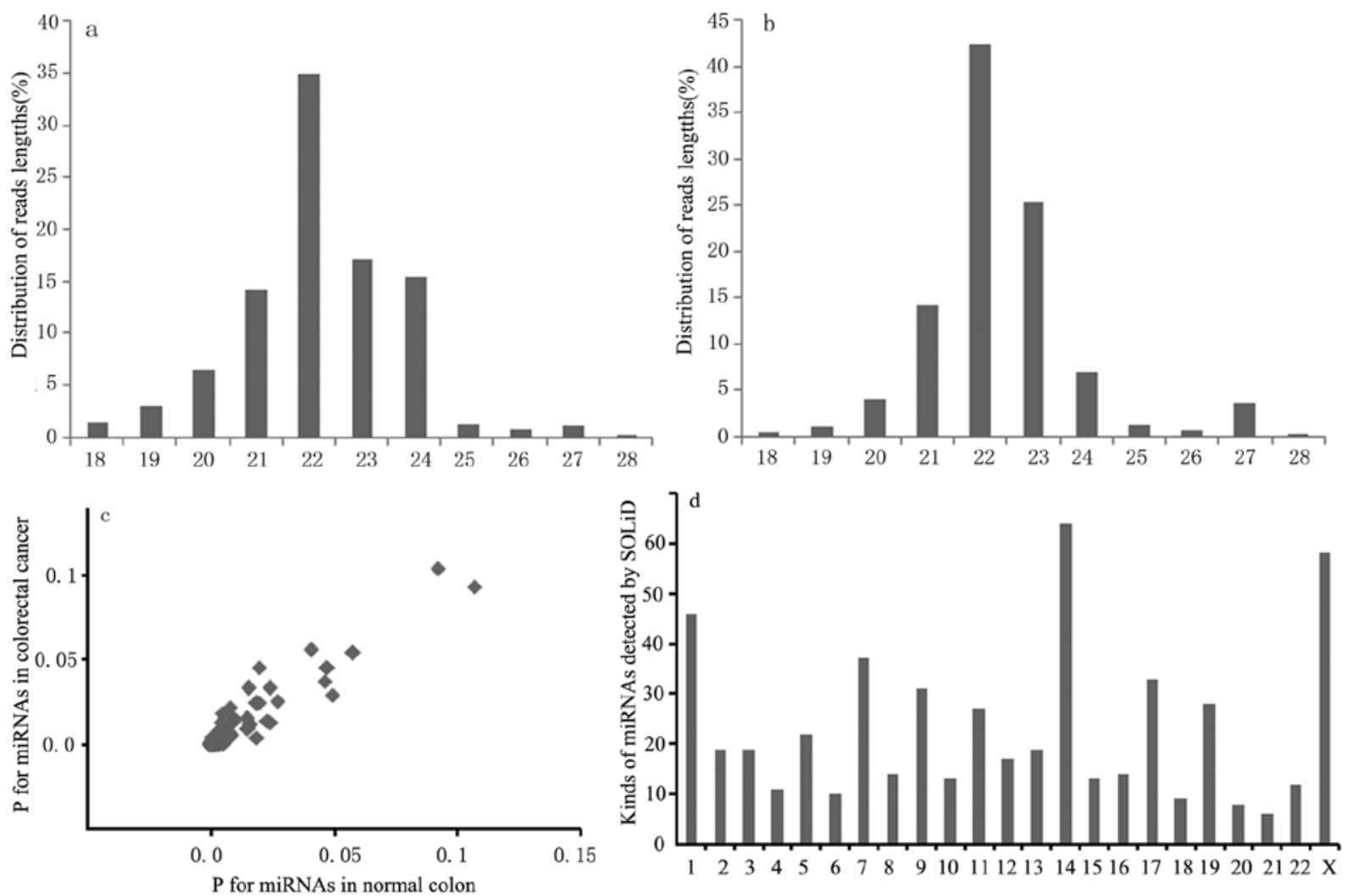

Figure 1. Results of SOLiD sequencing. (a) and (b), Reads length distribution of sequences obtained from SOLiD sequencing in normal colon and colorectal cancer, respectively. Sequences with 20-24 nucleotides (nt) length occupied the most part in all detected sequences. (c) Scatter diagram of relationship between miRNA coverage of the colorectal cancer and adjacent normal tissues. (d) Chromosome distribution of miRNAs revealed by SOLiD sequencing.

was used as a reference set (21). GO terms or KEGG pathway enrichment was determined by Z-test. In addition, enrichment analysis of signaling pathways, comparative analysis of biological functions and disease categories were performed to further explore functional characterization of the enriched target genes using DAVID bioinformatics tools (http://david. abcc.ncifcrf.gov). Finally, genes significantly affected were enriched to KEGG pathway through DAVID bioinformatics.

$$
p_{0 k}=\frac{\left[R_{m R N A k} \cdot N_{\text {total }}\right]_{\text {test }}+\left[R_{m R N A k} \cdot N_{\text {total }}\right]_{\text {control }}}{N_{\text {total test }}+N_{\text {total } \text { control }}}
$$

Statistical analysis. Z-test assay was used to investigate the differences in repression of targeted mRNAs in colorectal cancer (22). $P_{0}$ was calculated with formula 4 above, which was the estimate of the proportions whether the null hypothesis was true.

$$
Z_{k}=\frac{R_{m R N A k_{\text {test }}}-R_{m R N A k \text { control }}}{\sqrt{p_{0 k}\left(1-p_{0 k}\right) / N_{\text {total test }}+p_{0 k}\left(1-p_{0 k}\right) / N_{\text {total } \text { control }}}}
$$

When the Z-value $>1.96$ or $<-1.96$, the null hypothesis was rejected, which means that there were significant differences between the repression of mRNAs in the colorectal cancer versus adjacent normal tissues.

\section{Results}

Sequencing of the small RNA transcriptomes of 10 paired colorectal cancer samples. Small RNA transcriptomes from 10 colorectal cancer and 10 adjacent non-tumoral tissues were analyzed using the SOLiD sequencer. A total of 15141013 sequencing reads were obtained from the two group samples, their Read length distribution after adapter removal suggest that the majority of sequence were 20-24 nt (Fig. 1a and b), which indicates that mature miRNAs were enriched in the sequenced samples, and their proportion is $\sim 93.3 \%$. After removing the reads of other RNA (rRNA, tRNA and snRNA) and comparing with miRBase database, we obtained 627 and 451 miRNAs from total copies of several hundreds of thousands in colorectal cancer and adjacent normal tissues, respectively (available upon request).

The coverage of majority miRNAs $(80 \%)$ is $<0.001$, which indicate that the sensitivity and feasibility of SOLiD sequencing in recovering miRNAs with low coverage. Next, we analyzed the correlation between the coverage of miRNAs in colorectal cancer and adjacent normal tissues. Although correlation of some miRNAs partly deviated from one in colorectal cancer, most miRNAs are slight regulated (Fig. 1c), which is confirmed by our fold-change analysis. In addition, the distribution of different miRNAs in each chromosome were investigated, Fig. 1d shows that chromosome 14, chromosome $\mathrm{X}$ and chromosome 1 have relatively more distributed miRNAs.

Differentially expressed miRNAs in colorectal primary tumors. We analyzed the expression level of all detected miRNAs in the samples. Matches to known miRNAs in miRBase (release 15.0) represented $57.9 \%$ of the normal colon reads and $66.7 \%$ of colorectal cancer reads. A total of 673 known miRNAs was identified in two groups, with a greater 
a

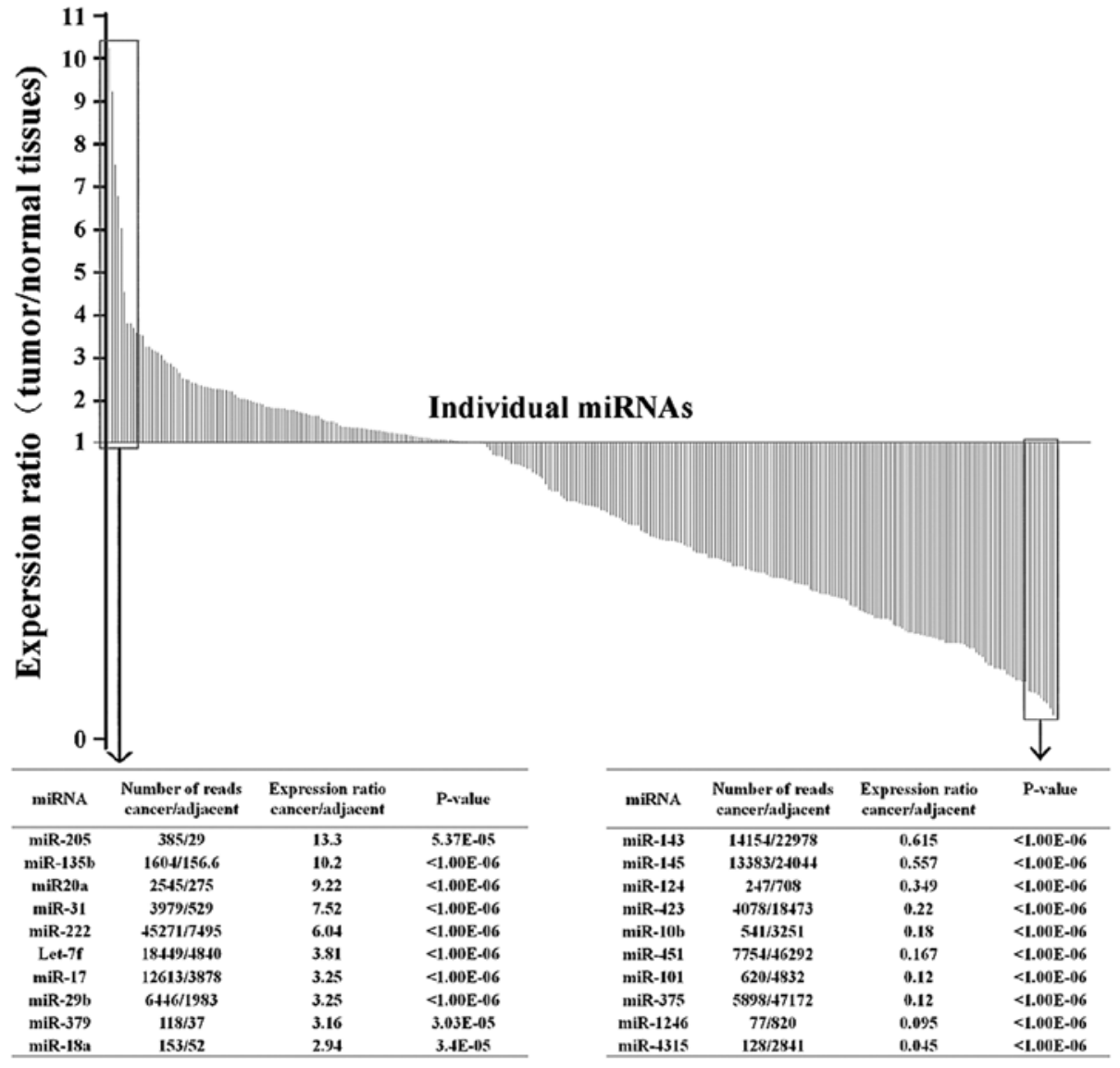

b

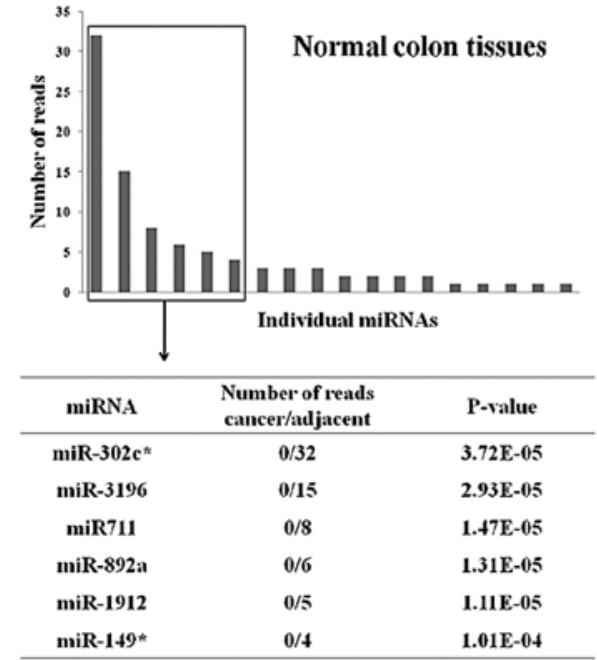

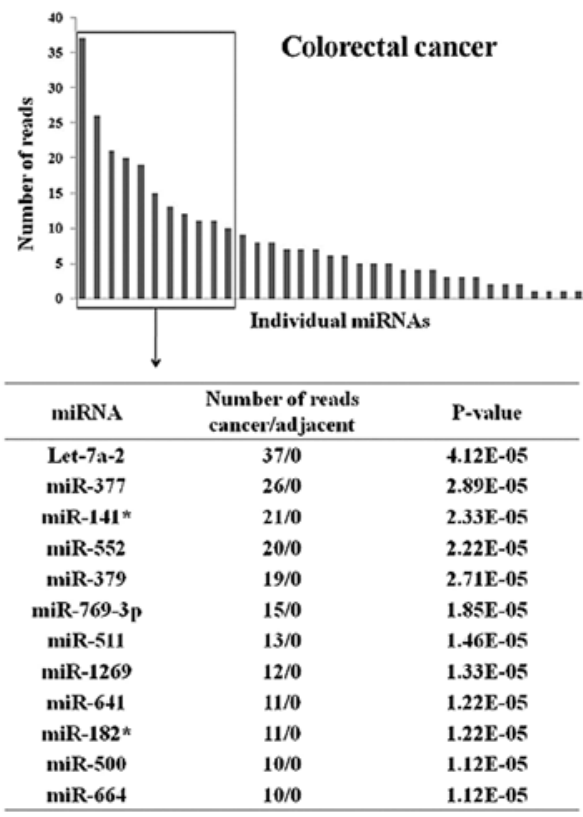

Figure 2. Differential expression of known miRNAs between colorectal cancer and adjacent normal tissues. (a) Expression ratios (percentage of total reads in colorectal cancer divided by percentage of total reads in adjacent normal tissues) are shown for all known miRNAs that were detected in both colorectal cancer and normal tissues datasets. Specific data pertaining to the 10 most differentially expressed miRNAs at both ends of the spectrum are displayed in the inset. (b) The absolute number of reads obtained for miRNAs that were detected solely in either colorectal cancer or in adjacent normal tissues.

total number of miRNAs expressed in colorectal cancer than in adjacent normal tissues (data not shown). An unsupervised analysis was performed after selection of the 313 miRNAs coexpressed in two group samples (Fig. 2a). There was a significant difference between non-tumor and tumor samples
$(\mathrm{P}<0.01)$. Of note, most of these miRNAs are associated with chromosomal regions of human tumor susceptibility loci that are known to have frequent gains or losses (23-25). Among them, a total of 36 significant differentially expressed miRNAs was identified. The 10 most up- and downregulated miRNAs 


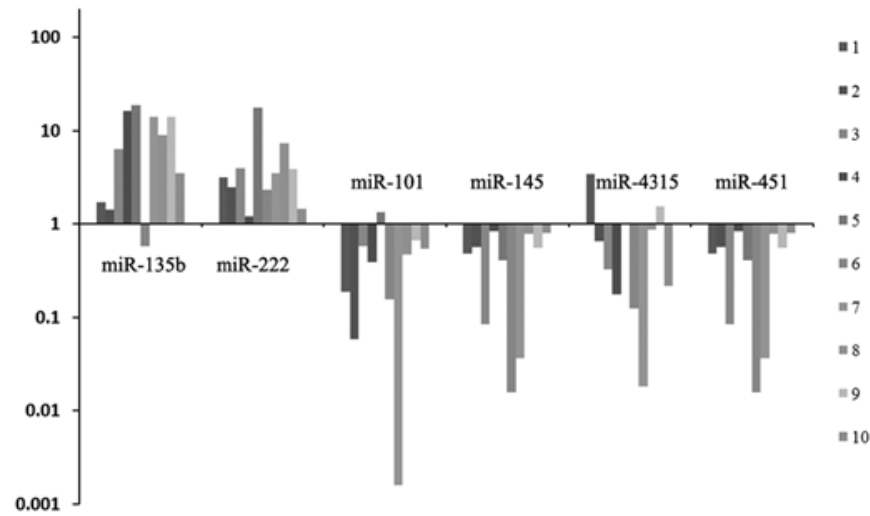

Figure 3. Deregulated miRNA expression in different colorectal cancer samples.

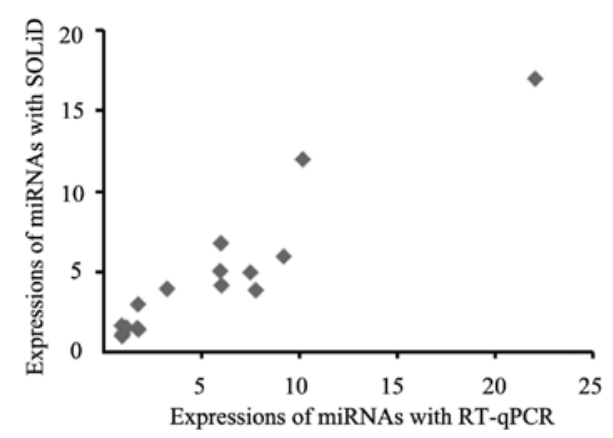

Figure 4. Correlation between expression ratios of miRNAs detected with SOLiD and qRT-PCR.

in colorectal cancer are shown in Fig. 2a. These miRNAs have 2 -fold overexpression and Fisher's exact test P-values $<0.01$. In addition, we surveyed the miRNAs that were expressed in normal colon tissues while diminish in colorectal cancer and that undetected in normal colon while expressed in colorectal cancer, as these miRNAs might be expected to participate in tumor-specific functions (Fig. 2b).

In addition, 16 significantly deregulated miRNAs were selected and validated by RT-qPCR. Further analysis of the 16 miRNAs shows that many were coordinately expressed, including the miR-17, miR-222, miR-101, miR-143, miR-145, miR-4315 and miR-17-92 clusters, which were consistently down- or upregulated in CRC (Fig. 2a). Among them, two miRNAs are significantly upregulated (miR-135b and miR-222) and four are downregulated (miR-101, miR-145, miR-4315 and mir-451) in majority of tumor samples (Fig. 3).

Correlation of miRNA sequencing to $R T-q P C R$ results. To verify the accuracy of SOLiD sequencing data of miRNAs, the qPCR result of the 16 selected differently expressed miRNAs were used to further analyze the correlation with the SOLiD data. The Pearson's correlation coefficient between sequencing and RT-qPCR data was calculated for 16 miRNAs across the 10 patients. The expression of the majority miRNAs is highly correlated between the two technical platforms (Fig. 4). This was also confirmed on the level of individual datasets, with Pearson's correlation coefficients ranging from 0.65 to 0.76 for each tumor (data not shown). The results suggest that normalized expression values obtained from miRNA deep
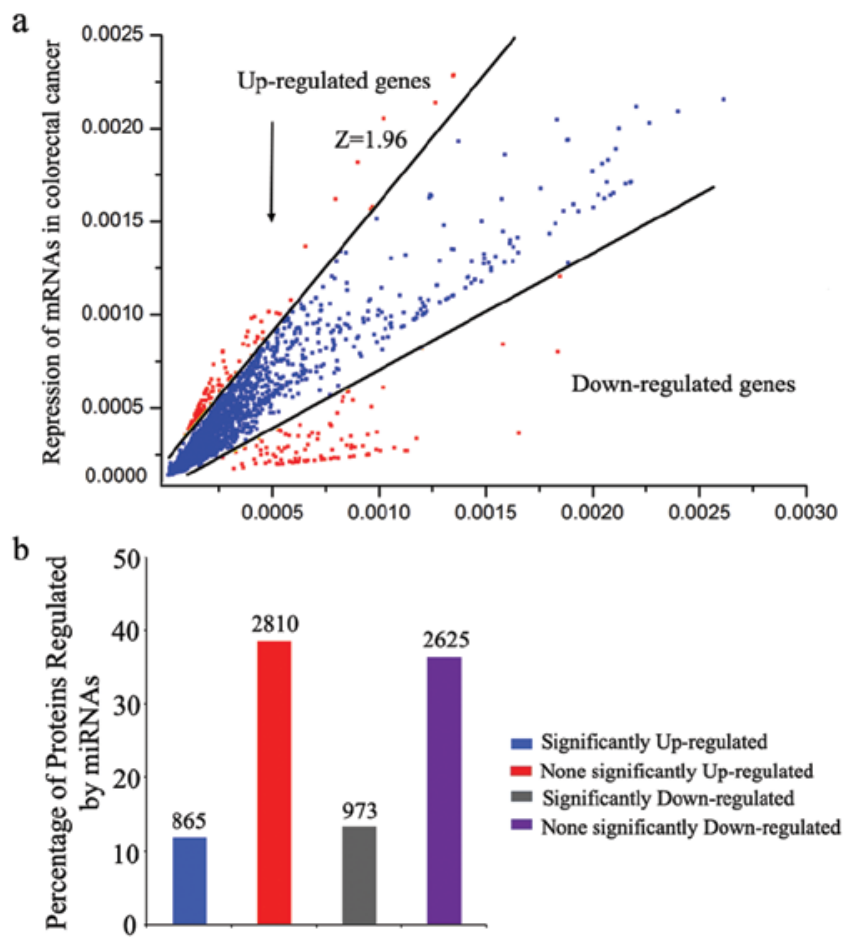

Figure 5. The distribution of genes regulated by miRNAs in colorectal cancer. (a) The proportion of differentially expressed genes in colorectal. (b) Distribution of proteins regulated by miRNAs.

sequencing are valid and comparable to RT-qPCR data, demonstrating that there was a very good correlation between the expression patterns of miRNAs obtained from the SOLiD sequencing and RT-qPCR. Therefore, the expression patterns of miRNAs detected by SOLiD sequencing could be feasible for evaluating relative expression of miRNAs.

Regulation of mRNAs by miRNAs in colorectal cancer. With the assay mentioned above, we gained repression degrees of miRNAs to their target mRNA in colorectal cancer (Fig. 5). Approximately 7273 mRNAs were repressed by miRNAs in colorectal cancer (available upon request). Subsequently, we performed the Z-test to compare the differences between the repression of miRNAs in colorectal cancer and adjacent normal tissues. The Z-test results indicated that many mRNAs were significantly repression by miRNAs in disease state (Fig. 5a). However, those mRNAs, including Ras, p53 and PTEN, significantly repressed by miRNAs were a minority of all targeted mRNAs.

A relatively small group of 1838 genes $(\sim 25 \%$ of total regulated genes), 865 upregulated and 973 downregulated, are significantly affected by the disease state (Fig. 5b). These results can help to understand the roles of miRNA in the pathogenesis of colorectal cancer.

KEGG and gene ontology for colorectal cancer enriched by miRNAs. Those genes significantly dysregulated in colorectal cancer were enriched into KEGG pathway through DAVID bioinformatics tools. We found that many KEGG pathways were significantly affected in colorectal cancer (Fig. 6). These pathways could be represented as main branches in KEGG pathway database. Among them, many were signal transduc- 

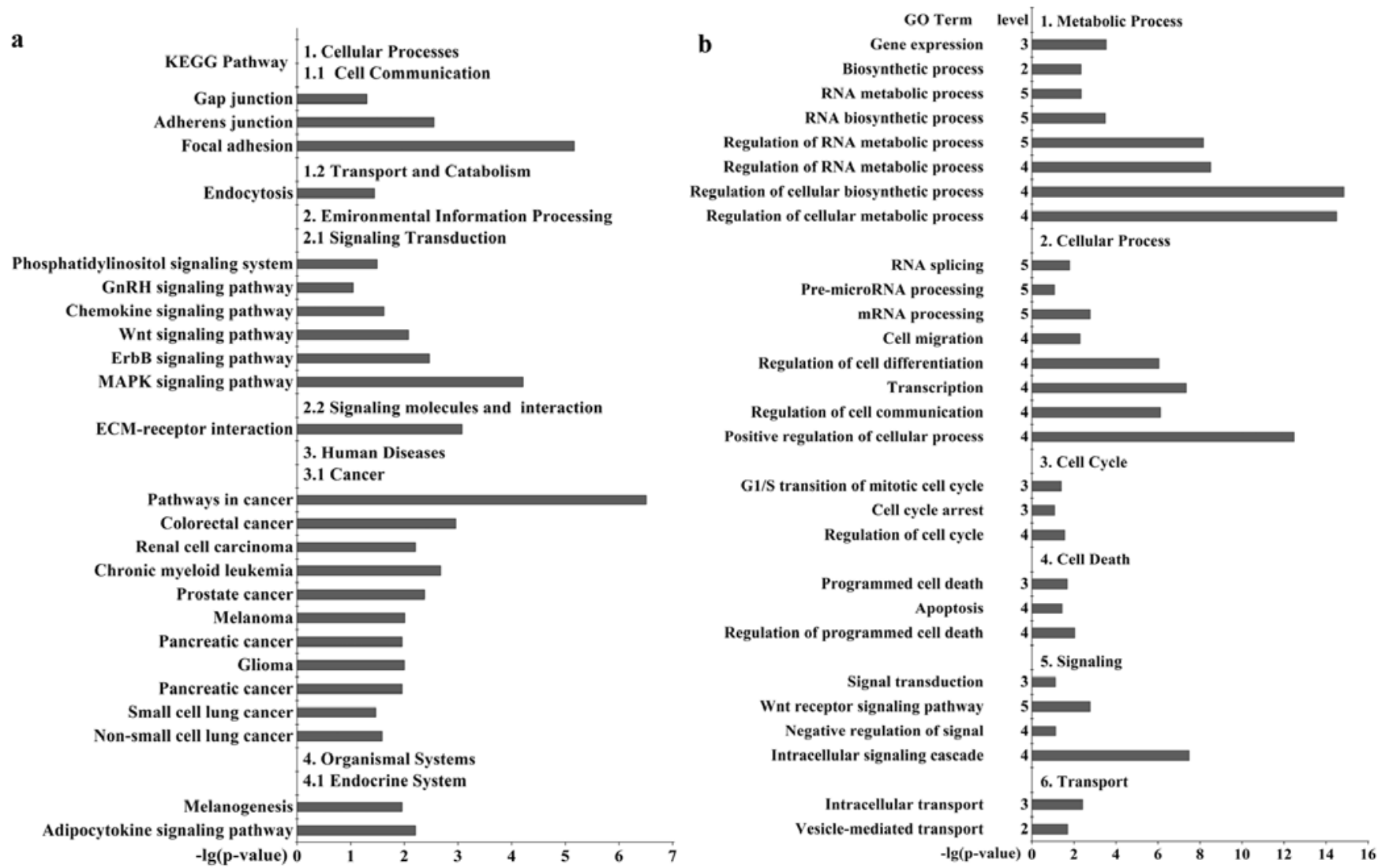

Figure 6. KEGG and GO for colorectal cancer enriched by miRNAs. (a) KEGG pathways significantly regulated in colorectal cancer. The significant level of 0.05 was applied in this study. (b) GO terms rooted to Biological Processes significantly regulated in colorectal cancer.

tion pathway that participate in cancer-related events (Fig. 6a), which suggested that the disease state could be amplified or declined by those signal transduction pathways.

As shown in Fig. 6a, among the significantly regulated KEGG pathway, two KEGG pathways rooted to cell communication (gap junction and adherens junction) and another two pathways rooted to signal transduction (Wnt signaling and MAPK signaling pathway) were affected in colorectal cancer, prompting cancer progress.

GO provides an ontology of defined terms representing gene product properties (26). Among the several covered domains by GO, the biological process, operations or sets of molecular events with a defined beginning and end, can relevantly describe the functioning of integrated genes. Based on the significantly regulated genes, the biological processes involved in the colorectal cancer were globally assessed with the web-based GO analysis tool, DAVID. In this manner, we investigated the molecular mechanism of colorectal cancer at the system level.

Among those significantly affected GO terms, most of them were rooted to the regulation of processes for colorectal cancer, which indicates that cells intend to adjust themselves to the environment change in different pathological conditions. Except for those GO terms rooted to regulation, the significantly regulated $\mathrm{GO}$ terms were roughly divided into six categories (metabolic process, cellular process, cell cycle, cell death, signaling and transport) to comprehensively analyze the pathological process. The results of DAVID analysis indicate that some GO terms rooted to the metabolic process are widely regulated in colorectal cancer. Among those GO terms, many terms are co-regulated in colorectal cancer, for example, five GO terms at level four (cellular metabolic process, regulation of biosynthesis process, cell differentiation, transcription and intracellular signaling cascade) are significantly regulated in colorectal cancer, which reflects the common cytological effects in response to disease state.

\section{Discussion}

In this study, we screened 10 paired clinical CRC samples for miRNA expression. According the results of deep sequencing, the expression level of miRNAs was widely affected (Fig. 2a). The expression of 36 miRNAs were found to be altered in CRC clinical specimens compared with normal colon and 6 of these miRNAs showed the consistent expression pattern in 10 paired CRC samples. The majority of these miRNAs, based on Chinese patients, are concordant with the results from groups, which were based on other ethnicities $(27,28)$. Such as miR-143, $-145,-21,-375,-378$ and -96 . However, it should be noted that, different from previous reports, miR-1246, -192 , -4305 and $-92 \mathrm{a}$ are unique in Chinese patients. Furthermore, miR-135b and miR-222 previously identified as overexpressed in colorectal cancer were also upregulated in 10 independent paired CRC samples. Noteworthy, overexpression of the two miRNAs was previously identified as important key factors to promote cell invasion or proliferation in various epithelial tumor types (16). Recently, Wong et al (29) identified miR-222 as highly expressed in hepatocellular carcinoma (HCC) that 
promotes cell migration and invasion. It was demonstrated that the overexpression of miR-222 is a common phenomenon in $\mathrm{HCC}$ and could confer metastatic potentials in $\mathrm{HCC}$ cells through activating AKT signaling $(30,31)$. Concomitant analysis of different tumor samples enabled us to associate deregulation of expression of miR-101, -145, -451 and -4315 with common early events in colon tumorigenesis. A lower expression of miR-145 was also previously described in human lung adenocarcinoma, and overexpression of miR-145 inhibits significantly cell proliferation by targeting EGFR and NUDT1 in A549 cells (32). These results enlightened the importance of refining classification based on molecular and meticulous characterization of the tumor phenotypes. Our observation that miR-145 and miR-451 were downregulated in CRC consistent with earlier studies (33). Therefore, the dysregulated expression of miRNAs could be a passive adaptive response to disease state.

A system approach to better understand the relationship of miRNA-mRNA is often used in order to gain insight into miRNA function. In previous studies of miRNA expression profiling obtained by deep sequencing, only the miRNAs with significant difference in expression were concerned while those miRNAs with high coverage but less significant difference were neglected (34). It results in an incomplete understanding of the complex mechanism of miRNA regulation. In this study, we attempted to make a more reliable annotation for the complex mechanism by considering all miRNAs with the model described above. In this manner, all miRNAs recovered by deep sequencing were firstly assigned equally to all their target mRNAs, and then multiplied by the weight factor for binding its target mRNAs, respectively, and the total coverage for a certain mRNA were then summed up from all types of miRNAs.

Since mRNA downregulation depends on the concentration of mRNA bound to the mRNA-miRNA complex, the degree of miRNA repression on its targeted mRNA can be determined by the coverage of mRNA-miRNA complexes. During the maturation of miRNAs, the rate of miRNA formation from double-stranded RNA is lower than that of miRNA-load RISC formation $(35,36)$, this indicates that the formation of miRNA-load RISC occurs simultaneously with the miRNA formation. The competitive interaction between targeted mRNAs of a certain miRNA suggests that miRNA-load RISC can be arrested by mRNAs to decoy their suppression mechanisms (37). By inducing the parameter RD, which present the suppression effect of miRNA, closely related functional mRNAs, as the target of miRNA, are predicted comprehensibly with our model.

With the model provided above, we evaluated all miRNAs recovered by deep sequencing between the two divergent clinical groups, and obtained repression degrees of miRNAs on their target mRNA in colorectal cancer (Fig. 5). These target mRNAs, negatively correlated to the miRNAs regulated, were commonly enriched in cancer-related pathways. The number of predicted targeted mRNA exceeds the number of their miRNA in colorectal cancer, illustrating the complexity of miRNA-mediated regulation on mRNA. Moreover, those mRNAs significantly repressed by miRNAs, such as ras, p53 and PTEN, are among the minority of targeted mRNAs, these results are consistent with previous studies (38-40).
These studies can help to understand the roles of miRNA in the pathogenesis of colorectal cancer.

The KEGG pathway database records networks of molecular interactions in the cells (41). Fig. 6 shows that many KEGG pathways were significantly affected in colorectal cancer. It also suggested that these signal transduction pathways amplified or declined the disease state, which are consistent with a previous study (25). Pathway analysis of these differentially expressed genes identified the G1/S cell cycle checkpoint, and Wnt signal pathways as significantly downregulated in CRC. This provided an explanation as to why a panel of miRNAs are deregulated in colorectal cancer. Therefore, miRNA deregulation is an adaptive process in pathological conditions.

The regulation of a cellular process is widely triggered by external and internal changes $(42,43)$, including the dysregulated expression of miRNA (44). Based on the mathematic model proposed in the study, the effects of miRNAs were systematic analyzed on mRNA level. The amount of mRNA could be globally regulated by miRNA in colorectal cancer, and the translation of some mRNAs was significantly regulated according to the Z-test. With in-depth analysis of miRNA mechanisms, the mathematic model could be modified to synthetically investigate the functions of miRNAs at the biological level of the system. Our method comprehensibly evaluated the complexity of miRNA-mediated regulation in gene expression.

In conclusion, by comparing tumors of divergent biology and clinical outcome, we provided insights into the heterogeneity of the small RNA transcriptomes in cancer. This study presents a more comprehensibly analysis on gene alterations to better understand CRC. Our study employed the miRNA expression signature to identify clinical and histological subtypes of tumors. The gene and protein networks directly targeted and affected by miRNAs that participate in tumorigenesis remain to be extensively explored. This led us to identify the consistently deregulated miRNA in colorectal cancer through miRNAome. Our results from gene ontology and KEGG analysis of predicted targets may contribute to establish the definitive genomic landscape and determine the role of miRNAs in disease. This allows us to better understand the molecular mechanism of colorectal cancer.

\section{Acknowledgements}

This study was financially supported by Natural Science Foundation of China (Nos. 61071047, 81071230, 31371003) and Natural Science Foundation of Jiangsu Province (No. BK2012122).

\section{References}

1. Weitz J, Koch M, Debus J, Höhler T, Galle PR and Büchler MW: Colorectal cancer. Lancet 365: 153-165, 2005.

2. Liefers GJ and Tollenaar R: Cancer genetics and their application to individualised medicine. Eur J Cancer 38: 872-879, 2002.

3. Fearon ER and Vogelstein B: A genetic model for colorectal tumorigenesis. Cell 61: 759-767, 1990.

4. Wood LD, Parsons DW, Jones S, et al: The genomic landscapes of human breast and colorectal cancers. Science 318: 1108-1113, 2007.

5. Alon U, Barkai N, Notterman DA, et al: Broad patterns of gene expression revealed by clustering analysis of tumor and normal colon tissues probed by oligonucleotide arrays. Proc Natl Acad Sci USA 96: 6745-6750, 1999. 
6. Williams NS, Gaynor RB, Scoggin S, et al: Identification and validation of genes involved in the pathogenesis of colorectal cancer using cDNA microarrays and RNA interference. Clin Cancer Res 9: 931-946, 2003

7. Lawrie CH: MicroRNAs and haematology: small molecules, big function. Br J Haematol 137: 503-512, 2007.

8. Wiemer EA: The role of microRNAs in cancer: No small matter. Eur J Cancer 43: 1529-1544, 2007.

9. Bartel DP: MicroRNAs: Genomics, biogenesis, mechanism, and function. Cell 116: 281-297, 2004.

10. Esquela-Kerscher A and Slack FJ: Oncomirs - microRNAs with a role in cancer. Nat Rev Cancer 6: 259-269, 2006.

11. Lewis BP, Burge CB and Bartel DP: Conserved seed pairing, often flanked by adenosines, indicates that thousands of human genes are microRNA targets. Cell 120: 15-20, 2005.

12. Akao Y, Nakagawa Y and Naoe T: MicroRNAs 143 and 145 are possible common onco-microRNAs in human cancers. Oncol Rep 16: 845-850, 2006

13. Schetter AJ, Leung SY, Sohn JJ, et al: MicroRNA expression profiles associated with prognosis and therapeutic outcome in colon adenocarcinoma. JAMA 299: 425-436, 2008.

14. Schepeler T, Reinert JT, Ostenfeld MS, et al: Diagnostic and prognostic microRNAs in stage II colon cancer. Cancer Res 68 : 6416-6424, 2008

15. Wang YX, Zhang XY, Zhang BF, Yang CQ, Chen XM and Gao HJ: Initial study of microRNA expression profiles of colonic cancer without lymph node metastasis. J Dig Dis 11 50-54, 2010 .

16. Nagel R, le Sage C, Diosdado B, et al: Regulation of the adenomatous polyposis coli gene by the miR-135 family in colorecta cancer. Cancer Res 68: 5795-5802, 2008.

17. Diosdado B, van de Wiel MA, Terhaar Sive Droste JS, et al MiR-17-92 cluster is associated with $13 \mathrm{q}$ gain and c-myc expression during colorectal adenoma to adenocarcinoma progression. Br J Cancer 101: 707-714, 2009.

18. Schulte JH, Marschall T, Martin M, et al: Deep sequencing reveals differential expression of microRNAs in favorable versus unfavorable neuroblastoma. Nucleic Acids Res 38: 5919-5928, 2010.

19. Li S, Wang H, Qi Y, et al: Assessment of nanomaterial cytotoxicity with SOLiD sequencing-based microRNA expression profiling. Biomaterials 34: 9021-9030, 2011.

20. Livak KJ and Schmittgen TD: Analysis of relative gene expression data using real-time quantitative PCR and the 2(-Delta Delta C(T)) method. Methods 25: 402-408, 2001.

21. Huang da W, Sherman BT, Tan Q, et al: DAVID Bioinformatics Resources: expanded annotation database and novel algorithms to better extract biology from large gene lists. Nucleic Acids Res 35: W169-W175, 2007.

22. Kal AJ, van Zonneveld AJ, Benes V, et al: Dynamics of gene expression revealed by comparison of serial analysis of gene expression transcript profiles from yeast grown on two different carbon sources. Mol Biol Cell 10: 1859-1872, 1999.

23. Staub E, Gröne J, Mennerich D, et al: A genome-wide map of aberrantly expressed chromosomal islands in colorectal cancer. Mol Cancer 5: 37, 2006.

24. Zhang L, Huang J, Yang N, et al: microRNAs exhibit high frequency genomic alterations in human cancer. Proc Natl Acad Sci USA 103: 9136-9141, 2006.

25. Calin GA, Sevignani C, Dan Dumitru C, et al: Human microRNA genes are frequently located at fragile sites and genomic regions involved in cancers. Proc Natl Acad Sci USA 101: 2999-3004, 2004.
26. Ashburner M, Ball CA, Blake JA, et al: Gene ontology: tool for the unification of biology. The Gene Ontology Consortium. Nat Genet 25: 25-29, 2000.

27. Callari M, Dugo M, Musella V, et al: Comparison of microarray platforms for measuring differential microRNA expression in paired normal/cancer colon tissues. PLoS One 7: e45105, 2012.

28. Drebber U, Lay M, Wedemeyer I, et al: Altered levels of the onco-microRNA 21 and the tumor-supressor microRNAs 143 and 145 in advanced rectal cancer indicate successful neoadjuvant chemoradiotherapy. Int J Oncol 39: 409-415, 2011.

29. Wong QW, Ching AK, Chan AW, et al: MiR-222 overexpression confers cell migratory advantages in hepatocellular carcinoma through enhancing AKT signaling. Clin Cancer Res 16: 867-875, 2010.

30. Pineau P, Volinia S, McJunkin K, et al: miR-221 overexpression contributes to liver tumorigenesis. Proc Natl Acad Sci USA 107: 264-269, 2010.

31. Ladeiro Y, Couchy G, Balabaud C, et al: MicroRNA profiling in hepatocellular tumors is associated with clinical features and oncogene/tumor suppressor gene mutations. Hepatology 47: 1955-1963, 2008

32. Cho WC, Chow AS and Au JS: MiR-145 inhibits cell proliferation of human lung adenocarcinoma by targeting EGFR and NUDT1. RNA Biol 8: 125-131, 2011.

33. Arndt GM, Dossey L, Cullen LM, et al: Characterization of global microRNA expression reveals oncogenic potential of miR-145 in metastatic colorectal cancer. BMC Cancer 9: 374 , 2009.

34. Tan KS, Armugam A, Sepramaniam S, et al: Expression profile of MicroRNAs in young stroke patients. PLoS One 4: e7689, 2009.

35. Kohler JJ and Schepartz A: Kinetic studies of Fos.Jun.DNA complex formation: DNA binding prior to dimerization. Biochemistry 40: 130-142, 2001

36. Bartlett DW and Davis ME: Insights into the kinetics of siRNA-mediated gene silencing from live-cell and live-animal bioluminescent imaging. Nucleic Acids Res 34: 322-333, 2006.

37. Poliseno L, Salmena L, Zhang J, Carver B, Haveman WJ and Pandolfi PP: A coding-independent function of gene and pseudogene mRNAs regulates tumour biology. Nature 465: 1033-1090, 2010

38. Stefanius K, Ylitalo L, Tuomisto A, et al: Frequent mutations of KRAS in addition to BRAF in colorectal serrated adenocarcinoma. Histopathology 58: 679-692, 2011.

39. Deschoolmeester V, Boeckx C, Baay M, et al: KRAS mutation detection and prognostic potential in sporadic colorectal cancer using high-resolution melting analysis. Br J Cancer 103: $1627-1636,2010$

40. Bouchahda M, Karaboué A, Saffroy R, et al: Acquired KRAS mutations during progression of colorectal cancer metastases: possible implications for therapy and prognosis. Cancer Chemother Pharmacol 66: 605-609, 2010.

41. Kanehisa M and Goto S: KEGG: kyoto encyclopedia of genes and genomes. Nucleic Acids Res 28: 27-30, 2000.

42. Gidron Y, De Zwaan M, Quint K and Ocker M: Influence of stress and health-behaviour on miRNA expression. Mol Med Rep 3: 455-457, 2010 .

43. Yu AM: Role of microRNAs in the regulation of drug metabolism and disposition. Expert Opin Drug Metab Toxicol 5: 1513-1528, 2009.

44. Li SC, Wang Y, Wang H, et al: MicroRNAs as participants in cytotoxicity of CdTe quantum dots in NIH/3T3 cells. Biomaterials 32: 3807-3814, 2011 